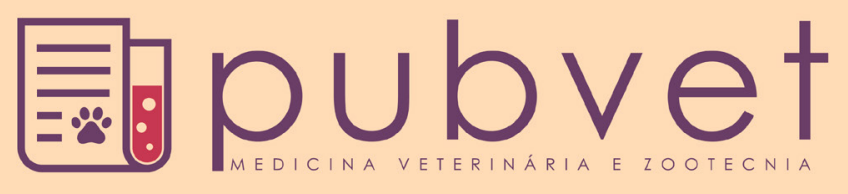

HTTP://DX.DOI.ORG/10.22256/PUBVET.V11N7.694-700

\title{
Megabacteriose em Calopsita (Nymphicus hollandicus)
}

\section{Laurien de Araujo Cavalcante Filho ${ }^{1}$, Júlio Cézar dos Santos Nascimento ${ }^{2 *}$, Lucilo Bioni da Fonseca Filho ${ }^{3}$, Marleyne José Afonso Accioly Lins Amorim ${ }^{4}$, Mercia Rodrigues Barros $^{5}$, Roseana Tereza Diniz de Moura ${ }^{6}$}

\author{
${ }^{1}$ Médico Veterinário e Zootecnista. E-mail: laurienzootec@gmail.com \\ ${ }^{2}$ Professor do Centro Universitário Maurício de Nassau, Curso de Medicina Veterinária, Recife-PE Brasil. E-mail: \\ juliocezar@veterinario.med.br \\ ${ }^{3}$ Acadêmico de Medicina Veterinária, Departamento de Medicina Veterinária, Universidade Federal Rural de Pernambuco, Recife-PE Brasil. \\ E-mail: lucilofilho@gmail.com \\ ${ }^{4}$ Professor Associado da Universidade Federal Rural de Pernambuco, Departamento de Morfologia e Fisiologia Animal, Recife-PE Brasil- \\ E-mail:mjaamorim@yahoo.com.br \\ ${ }^{5}$ Professor em Universidade Federal Rural de Pernambuco, Departamento de Medicina Veterinária, Recife-PE Brasil. E-mail: \\ merciarbpe@gmail.com \\ ${ }^{6}$ Professor em Universidade Federal Rural de Pernambuco, Departamento de Medicina Veterinária, Recife-PE Brasil. E-mail: \\ roseana.diniz@gmail.com \\ *Autor para correspondência
}

RESUMO. Macrorhabdus ornithogaster, agente das Megabacterioses de forma leveduriforme, é encontrado na microbiota normal do proventrículo de aves. Sob circunstâncias inespecíficas, o equilíbrio hospedeiro-fungo é rompido e os sinais clínicos, chamados "Síndrome Light Going", se manifestam. M. ornithogaster tem sido diagnosticado em laboratório como causa de doença em uma ampla diversidade de espécies de aves, com prognóstico reservado a depender do caso. Este trabalho aborda uma revisão de literatura sobre a megabacteriose em aves e relata um caso clínico em Calopsita (Nymphicus hollandicus), macho, de raça Cara Branca, com 1 ano de idade, onde se deu entrada na Clínica Veterinária. Por meio do histórico, anamnese, sinais clínicos, exame direto das fezes, chegou-se ao diagnóstico presuntivo da megabacteriose. Para o tratamento foi prescrito Nistatina $100.000 \mathrm{UL} / \mathrm{mL} /$ por via oral na dose de $0,4 \mathrm{~mL}$ duas vezes ao dia, por 15 dias via sonda de alimentação acoplada à seringa de insulina, juntamente com uma gota de Tilosina e uma gota de Glicopan Gold em duas tomadas diárias (dose diária recomendada pelo fabricante $1 \mathrm{~mL}$ ou 11 gts diluídos em $100 \mathrm{~mL}$ de água ou 2 a 3 gotas, uma vez ao dia) diretamente no bico, além de 5 gotas de vinagre de maçã em $100 \mathrm{~mL}$ de água para beber. Após 7 dias do tratamento, houve melhora do animal, mas ainda apresentava emagrecimento e sementes nas fezes. O tratamento seguiu e com 16 dias de tratamento o animal foi reavaliado, apresentando coloração normal das fezes, ganho de peso, ausência de regurgitação e sementes nas fezes, e voltou a cantar e recebeu alta.

Palavras chave: Proventriculite, micose, síndrome light going

\section{Megabacteriosis in Nymphicus hollandicus}

ABSTRACT. Macrorhabdus ornithogaster, agent of yeast-like Megabacteriosis is found in the normal microbiota of the proventriculus of birds. Under non-specific conditions, the host-fungal balance is disrupted and clinical signs, called "Light Going Syndrome", manifest. M. ornithogaster has been diagnosed in the laboratory as a cause of disease in a wide diversity of bird species, with a prognosis reserved to depend on the case. This work presents a review of the literature on megabacteriosis in birds and reports a clinical case in Calopsita (Nymphicus hollandicus), a 1 year old Cara White male, where he was admitted to the Veterinary Clinic. Through history, anamnesis, clinical signs, direct examination of feces, the presumptive diagnosis of megabacteriosis was reached. For the treatment 
Nystatin 100,000 IU/mL / oral (VO) was prescribed at $0.4 \mathrm{~mL}$ twice daily (Bid), for 15 days via a feeding tube attached to the insulin syringe, together with a drop of Tylosin and one drop of Glycopan Gold in two daily intakes (daily dose recommended by the manufacturer $1 \mathrm{~mL}$ or 11 gts diluted in $100 \mathrm{~mL}$ of water or 2 to 3 drops, once daily) directly in the beak, in addition to 5 drops of apple cider vinegar in $100 \mathrm{~mL}$ of drinking water. After 7 days of treatment, there was improvement of the animal, but still presented slimming and seeds in the faeces. The treatment was followed and with 16 days of treatment the animal was revaluated, presenting normal stool coloration, weight gain, absence of regurgitation and seeds in the faeces, and again singing and receiving discharge.

Keywords: Proventriculitis, mycosis, syndrome light going

\section{Proventriculitis infecciosa en Periquito (Nymphicus hollandicus)}

RESUMEN. Macrorhabdus ornithogaster, agente causante de la Megabacteriosis (proventrtriculitis infecciosa), hace parte de la microbiota normal del proventrículo de las aves de corral. Bajo circunstancias inespecíficas, el equilibrio huésped-hongo se quiebra y los signos clínicos, llamados " Síndrome Light Going", se manifiestan. M. Ornithogaster ha sido diagnosticado en laboratorio como causa de enfermedad en una amplia diversidad de especies de aves, con pronóstico reservado dependiendo del caso. Este trabajo aborda una revisión de literatura sobre megabacteriosis en pájaros y divulga el caso clínico de un periquito (Nymphicus hollandicus), macho, cara branca, con un año de edad, dando entrada para consulta en la clínica veterinaria. Por medio de su histórico, anamnesis, signos clínicos e examen directo de las heces, se llegó al diagnóstico presuntivo de megabacteriosis. Para el tratamiento se prescribió nistatina $100.000 \mathrm{IU} / \mathrm{mL} /$ oral en la dosis de 0,4 dos veces al día, durante 15 días a través de sonda de alimentación acoplada a la jeringa de insulina, junto con una gota de Tilosina y una gota de Glicopan Gold en dos tomadas diarias (dosis diaria recomendada por el fabricante $1 \mathrm{ml}$ o 11 GTS diluido en $100 \mathrm{ml}$ de agua o 2 a 3 gotas, una vez al día) directamente en el pico, más 5 gotas de vinagre de manzana en 100 $\mathrm{ml}$ de agua para beber. Después de 7 días del tratamiento, había mejora del animal, pero todavía presentando delgadez y semillas en las heces. El tratamiento seguido y con más 16 días de tratamiento se reevaluó el animal, presentando coloración normal de las heces, aumento de peso, ausencia de regurgitación, ausencia de semillas en las heces, volviendo a cantar y recibió alta.

Palabras clave: Proventriculitis, tiña, síndrome Light Going

\section{Introdução}

Tem-se observado um crescente número de casos em aves nas clínicas veterinária, acompanhado do aumento de casos de megabacteriose em aves na região metropolitana do Recife de Pernambuco. Esta afecção tem sido relatada em alguns países e em diversas espécies de aves, como periquito (Melopsittacus undulatus), canário (Serinus canarius), ema (Rhea americana), avestruz (Struthio camelus), mandarim (Taeniopygia guttata), tucano (espécie Ramphastidae), pomba (espécie Columbidae), codorna japonesa (Coturnix coturnix japonica), galinha (Gallus gallus) e peru (Meleagris gallopavo) (Queirós et al., 2011).

A megabacteriose é uma patologia de alta morbidade e mortalidade bastante variável, podendo chegar a $100 \%$ no lote em animais jovens e imunossuprimidos. Para o diagnóstico da infecção, baseia-se em histórico, anamnese, sinais clínicos, microscopia direta de impressão fresca da mucosa de ventrículo ou proventrículo, cultura de amostras de mucosa ventricular e proventricular, exame histopatológico, necropsia e presença do agente nas fezes.

Objetivou-se, com o presente trabalho relatar um caso clínico de megabacteriose em Calopsita, assim como apresentar uma revisão de literatura fornecendo um aporte de maior de informações sobre esta patologia subdiagnosticada na clínica médica de aves.

A megabacteriose, também chamada "Síndrome Light Going" é uma patologia que afeta aves de muitas espécies, tendo sido associada a uma condição crônica sintomática ou assintomática (Carvalho et al., 2011). 
Macrorhabdus ornithogaster é uma levedura ascomiceto anamórfica. Colonizam a junção estreita (istmo) do estômago glandular (proventriculus) e moagem de estômago (ventriculus) de aves. Em esfregaços das mucosas e das fezes, o microrganismo é uma haste rígida, com frente que é de $20 \mu \mathrm{m}$ a $80 \mu \mathrm{m}$ de comprimento e de 2 a $3 \mu \mathrm{m}$ de largura, com extremidades arredondadas (Phalen, 2007).

Segundo Phalen (2014) pensou-se ser uma bactéria (megabactéria) por mais de 20 anos. Werther et al. (2000) descreveram o microrganismo pela primeira vez como estruturas semelhantes a um fungo, em proventrículo de canários e periquitos. Posteriormente, $\underline{\text { Scanlan and }}$ Graham (1990) caracterizaram o agente como uma bactéria bacilar, gram positiva, PAS (Ácido Periódico de Schiff) positiva, de dimensões grandes, variando entre 1,5 a 3,0 $\mu \mathrm{m}$ de largura e 20 a $50 \mu \mathrm{m}$ de comprimento. Também sugeriram que o mesmo seria um componente normal da flora gastrointestinal de periquitos, desde que os microrganismos foram também isolados de aves saudáveis. Os autores referem que o fungo pode ser encontrado nas fezes e no muco do istmo entre proventrículo e ventrículo, e não se sabe sobre a resistência do agente no ambiente (Martins et al., 2006). Munhoz et al. (2008) observaram que o $M$. ornithogaster é um microrganismo oportunista, promovendo maior mortalidade na desordem imunossupressora.

A principal fonte de infecção do Macrorhabdus ornithogaster são as aves portadoras assintomáticas, não há indicações científicas que demonstrem haver transmissão vertical da megabactéria, no entanto, a alimentação dos filhotes através da regurgitação bem como a contaminação oro-fecal são as formas mais comuns de transmissão do agente. Da mesma forma, o alojamento conjunto de espécies diferentes unido de precária biossegurança aumentam as chances de transmissão do agente, fato este sustentado pela ocorrência de megabacteriose em canários e mandarins de um mesmo criatório, e galináceos e codornas japonesas alojadas em um mesmo galpão. $\mathrm{O}$ carácter oportunista do fungo pode ser evidenciado quando a infecção ocorre em associação com outras doenças, tais como coccidiose em tucanos, vírus de marek em frangos, candidíase em galinhas e tricomoníase em pombos, agravando as taxas de morbidade e mortalidade nos plantéis (Carvalho et al., 2011). Segundo Lanzarot et al. (2013), embora formas agudas de megabacteriose tenham sido descritas, principalmente em periquitos, o curso da doença é normalmente crônica, por vezes, com períodos intermitentes de recuperação e recaída. erca de um terço de periquitos saudáveis poderia apresentar $M$. ornithogaster no estômago, sem quaisquer sinais clínicos óbvios (Filippich et al., 1993, Fraga et al., 2011).

Segundo Phalen (2014), os sinais gastrointestinais mais habituais são diarreia, êmese, melena, associados ou não à progressiva perda de peso, caquexia, anorexia ou normorexia. Ainda há relatos de desuniformidade do lote, depressão, fraqueza, letargia, problemas de empenamento, piora da eficiência alimentar, além de atrofia dos músculos peitorais, acúmulo de fezes ao redor da cloaca, palidez de mucosas, queda da produção de ovos e baixo ganho de peso.

Sinais clínicos em aves de gaiola incluem perda de peso, êmese, diarréia, sementes inteiras nos excrementos e letargia. Experimentalmente aves infectadas mostraram uma taxa reduzida de ganho de peso, mas não há sinais clínicos de doença. Frangos de corte com infecções naturais, demostraram uma variedade de sinais, mas todos os lotes foram impactados por uma série de agentes de doenças e não era possível determinar se qualquer um dos sinais foram causados por $M$. ornithogaster (Filippich et al., 1993).

De acordo com Phalen (2014), o diagnóstico da infecção baseia-se em histórico, anamnese, sinais clínicos, microscopia direta de impressão fresca da mucosa de ventrículo ou proventrículo, cultura de amostras de mucosa ventricular e proventricular, em histopatológico, necropsia e presença do agente nas fezes. A megabacteriose pode causar um aumento do $\mathrm{pH}$ do muco proventricular de 2,7 para valores que podem variar de 7,0 a 7,3, por conseguinte, a medição de $\mathrm{pH}$ por meio de lavagem do proventrículo, também pode ajudar no diagnóstico (Werther et al., 2000). A necropsia revela frequentemente um pássaro magro com grandes lesões na junção entre o proventrículo e ventrículo. As lesões macroscópicas incluem uma proventriculite com úlceras com ou sem hemorragia, a dilatação do istmo gástrica, afinamento da parede do istmo e secreção de muco aumentada (Hoppes, 2012).

Conforme Phalen (2014), a ausência de $M$ ornithogaster nas fezes não exclui totalmente a infecção ou infecção e doença associada. Uma maneira rápida de se concentrar o fungo é separando fezes sólidas e homogeneizar com 
água, e cerca de 20 vezes o seu volume de soro fisiológico, em um pequeno tubo, deixando descansar por 10 segundos e, em seguida, examinar uma pequena gota da suspensão recolhida a partir da superfície. Um ensaio de reação em cadeia da polimerase (PCR) para detectar $M$ ornithogaster nas fezes também está disponível na América do Norte (Veterinary Molecular Diagnostics, Milford, Ohio, EUA).

Quando encontrados nas fezes, são longo, retos, hastes rígidas, com extremidades arredondadas. Em algumas circunstâncias, as longas varas podem curvar-se ligeiramente. Organismos em forma de $\mathrm{Y}$ podem ser vistos, mas estes são extremamente raros. Visualizados diretamente em uma solução, estruturas pequenas encontradas em intervalos regulares são vistas. Estas estruturas são núcleos. A coloração dos núcleos foi de acordo com Phalen (2014).

No exame post mortem, preparação salina da raspagem da junção (istmo) do proventrículo irá demonstrar os organismos abundantes. Em periquitos e pássaros com doença, encontra-se a mucosa engrossada do proventrículo e aumento de muco no lúmen, e alguns exemplares apresentam úlceras com sangue no proventrículo (Phalen, 2014).

Segundo Madani et al. (2014), o controle e tratamento da infecção do M. ornithogaster são um desafio para os clínicos. As infecções subclínicas podem ocorrer em muitas aves sem sinais clínicos evidentes, é quase impossível manter grandes aviários livre da infecção. Por outro lado, tem sido relatado que muitos fármacos antimicrobianos e antifúngicos como preparações de iodo, lufenuron, nistatina, fluconazol, cetoconazol, itraconazol e terbinafina não são eficazes no tratamento da infecção.

A anfotericina $\mathrm{B}$, nistatina e produtos químicos antifúngicos de baixa toxicidade (por exemplo, benzoato de sódio) têm algum grau de eficácia contra M. ornithogaster. Verificou-se ser eficaz no tratamento de galinhasquando administrados 100 $\mathrm{mg} / \mathrm{kg}$ por via oral duas vezes por dia durante 14 dias. A nistatina foi eficaz quando administrado em água a 3.500,000 UI/L de água potável durante 2 dias, em seguida, 2.000.000 UI por litro, durante 28 dias (Kheirandish and Salehi, 2011). O estudo foi bem controlado e, após o tratamento, um lote de pássaros foi eutanasiado e comprovou-se ser livre de infecção. Há algumas evidências que sugerem que algumas estirpes de $M$. ornithogaster são resistentes a nistatina ou a anfotericina $\mathrm{B}$
(Filippich and Perry, 1993). O benzoato de sódio, benzoato de potássio e sorbato de sódio podem prevenir o M. ornithogaster crescer in vitro (Bradley et al., 2005).

O tratamento mais eficaz foi relatado como sendo a anfotericina $\mathrm{B}$, a qual é dada por via oral ou por sonda alimentação a uma dose de 100 $\mathrm{mg} / \mathrm{kg}$ duas vezes por dia durante um mês. Uma preparação solúvel em água de anfotericina $\mathrm{B}$, quando administrada por 14 dias em água de beber, não foi eficaz. Estirpes de M. ornithogaster resistentes à anfotericina $\mathrm{B}$ foram identificadas na Austrália (Madani et al., 2014).

\section{Material e Métodos}

No dia 20 de fevereiro de 2016, deu entrada na Clínica Veterinária um psitacídeo da espécie calopsita, macho, de raça Cara Branca, pesando 80 gramas, um ano de idade, de nome Malhado. $\mathrm{O}$ tutor relatou que no primeiro dia observou o animal pouco ativo, sem cantar, penas eriçadas, polifagia, dormindo bastante. No dia seguinte, seguiu-se a observação dos mesmos sinais, como também apresentava diarreia forte, no terceiro dia, houve a presença de sementes não digeridas no forro da gaiola junto às fezes.

No histórico, foi relatado que a ave havia mudado de local onde se pode ter acesso de pardais em sua gaiola para se alimentar. Esse acesso gerava muito estresse à ave devido à defesa de território. Tutor relatou, ainda, que a ave regurgitava sementes inteiras.

No exame físico foi observado que o paciente se apresentava com mucosas hipocoradas, normotérmico, atrofia dos músculos peitorais (peito seco) e penas, ao redor da cloaca, com fezes aquosas. Coletou-se amostra de fezes para realizar um esfregaço. A amostra foi diluída com soro fisiológico no volume de 20 vezes o da amostra, homogeneizada e utilizaram-se duas gotas da solução em lâmina para observação em microscópio. Na observação da lâmina, havia hastes de Macrorhabdus ornithogaster (fungo), confirmando a suspeita de acordo com os sinais clínicos relatados.

Recomendou-se, ao tutor, a higienização da gaiola, comedouros e bebedouros, e utilização de folhas de papel, exceto jornal para evitar intoxicação por chumbo contido na tinta. Em relação à alimentação, suspender a ração comercial formulada e utilizar ração prescrita nas proporções adequadas, devido a relatos de rações mofadas e mal acondicionadas. 
Para o tratamento, foi prescrito Nistatina $100.000 \mathrm{UI} / \mathrm{mL} /$ por via oral (VO) na dose de 0,4 $\mathrm{mL}$ duas vezes ao dia (Bid), por 15 dias via sonda de alimentação acoplada à seringa de insulina, juntamente com uma gota (gt) de Tilosina e uma gota de Glicopan Gold em duas tomadas diárias (dose diária recomendada pelo fabricante $1 \mathrm{~mL}$ ou 11 gotas diluídos em $100 \mathrm{~mL}$ de água ou 2 a 3 gotas, uma vez ao dia) diretamente no bico, além de 5 gotas de vinagre de maçã em $100 \mathrm{~mL}$ de água para beber.

A Nistatina foi recomendada ao invés de Anfotericina B, devido ao fato do tutor já ter a medicação em casa. Ainda prescrita a Tilosina, um antibiótico de largo espectro.

Após sete dias de tratamento, o tutor relatou evolução do quadro clínico do animal, mas ainda apresentava emagrecimento e sementes nas fezes, e estava com coloração verde claro. O tratamento seguiu durante dezesseis dias e o animal foi reavaliado, apresentando coloração normal das fezes, ganho de peso, ausência de regurgitação e sementes nas fezes, e voltou a cantar. $\mathrm{O}$ animal então recebeu alta com peso de 112 gramas. Posteriormente ao tratamento, tentou entrar em contato com o tutor para uma nova coleta para verificar a presença do patógeno nas fezes, mas o mesmo não se encontrava em seu domicílio.

\section{Resultados e Discussão}

O exame das fezes é de crucial importância para o diagnóstico preciso e servir de diferencial com outras enfermidades que apresentam sinais clínicos semelhantes. Segundo Meirelles et al. (2015), a não visualização do agente não exclui a infecção, pois as aves infectadas podem eliminar o agente intermitentemente. Neste trabalho, 12 de 123 amostras de fezes examinadas $(9,75 \%)$ foram positivas. Todavia, decorrente de sua eliminação intermitente, resultados negativos não devem ser prontamente desconsiderados. $\mathrm{O}$ diagnóstico definitivo de $M$. ornithogaster é mais frequentemente demonstrado na necropsia (Hoppes, 2012).

Phalen (2014) relata que uso de Mini-FLOTAC é proposto como uma nova ferramenta rápida para a detecção de $M$. ornithogaster em aves vivas. Ensaios de reação em cadeia da polimerase (PCR) para detectar $M$ ornithogaster nas fezes está disponível só na América do Norte, restringindo seu uso. Dentre os exames clínicos possíveis de diagnóstico, o esfregaço de fezes foi o escolhido, neste relato de caso, justificado pelo seu baixo custo, diagnóstico imediato, possível visualização do fungo sem uso de corantes. Todas as características observadas se enquadravam com o curso agudo da megabacteriose.

Não se pode descartar, em psitacídeos, a ocorrência de uma ou duas infecções fúngicas associadas e descritas em Melopsittacus undulatus (Filippich and Hendrikz, 1998), Amazona aestiva (Carrasco et al., 1998), Cacatua sulphurea sulphurea (Anderson, 1993), Nymphicus hollandicus (Vasconcelos et al., 2011), Agapornis fischeri (Nouri and Kamyabi, 2010). Não foram descritos ainda no Brasil relatos da ocorrência associada de aspergilose, megabacteriose e candidíase em Melopsittacus undulates. Isso demonstra a necessidade de uma atenção maior dos médicos veterinários em relação a pesquisas de doenças fúngicas em aves e suas associações.

Visto como microrganismo normal da microbiota intestinal de aves, ou como um agente oportunista de distribuição mundial, Candida spp., principalmente a espécie Candida albicans, provoca micoses endógenas que acometem principalmente o trato digestivo superior das ave. Em geral psitacídeos são as principais espécies acometidas por candidíases, apresentando-se deprimidos, com esvaziamento gástrico retardado, regurgitação, inapetência e dificuldade na digestão dos alimentos (Silva et al., 2014).

O esvaziamento gástrico retardado não é mensionado pelos autores como sinal clínico na megabacteriose, podendo direcionar um diagnóstico presuntivo para a megabactéria. $\mathrm{O}$ diagnóstico precoce dessas infecções consiste em um desafio para tratamento e controle (Dahlhausen, 2006).

A associação de falhas nutricionais, estresse, debilidade, imunossupressão, diminuição de ventilação, mudanças de temperatura, falhas de higienização, ambiente inapropriado, antibioticoterapia prolongada, administração de corticóides e traumatismos predispõem ao surgimento de infecções fúngicas em aves ( $\underline{\text { Fraga, }}$ 2014). As infecções fúngicas estão normalmente associadas à deficiência imunológica, estresse, desnutrição e condições precárias de higiene (Cubas et al., 2014).

Com o propósito de um menor estresse à ave, os medicamentos foram administrados em conjunto, nistatina, tilosina e nutracêutico Glicopan Gold via oral. A administração dos medicamentos através da sonda proporciona um 
controle melhor do tratamendo, em comparação à medicação na água. Filippich et al. (1993) relatam que os medicamentos diluídos na água de beber, afirmam que a taxa de dosagem na ave tratada vai depender da quantia de água ingerida, e algumas espécies de aves bebem pouca água, já outras apresentam depressão, sem haver consumo desejável do medicamento.

A administração de nistatina suspensão $(100.000 \mathrm{UI} / \mathrm{mL} / \mathrm{VO}$ na sonda, $1 \mathrm{~mL} / 300$ gramas $(\mathrm{g}) / \mathrm{Bid} / 14$ dias) corrobora com Cubas et al. (2014). Historicamente os únicos tratamentos eficazes têm sido anfotericina $\mathrm{B}$ e nistatina,esta última tem sido relatada como sendo eficaz em pintassilgos. A anfotericina B é mais eficaz em aves se administrado por via oral, quer em água medicada ou por gavagem (alimentação forçada). Embora a anfotericina B possa ser eficaz, seu custo elevado torna seu uso inapropriado em grandes plantéis (Hoppes, 2012). Segundo Dahlhausen (2006) a anfotericina B é eliminada pelos rins e é usado somente em curta duração devido ao risco de nefrotoxicidade induzida, e deve ser usada em combinação com itraconazol ou fluconazol.

\section{Considerações Finais}

A megabacteriose é uma enfermidade cada vez mais comum na clínica de animais silvestres, ornamentais e de produção e reprodução, e a quantidade de óbitos é significativa, devido ao curso hiperagudo da doença (em horas) e à dificuldade do diagnóstico diferencial, em relação ao das bacterioses. Na clínica de aves, constitui um desafio a conscientização dos tutores que fazem seu próprio diagnóstico e tratamento, recomendado por balconistas: "Toda diarreia é verminose". O simples procedimento de exame fecal pode fazer uma grande diferença na hora de se decidir que terapia instituir mediante sinais clínicos como diarreia. Conhecer a epidemiologia, patologia, espécies acometidas e eficiência de tratamentos da megabacteriose em aves são temas ainda restrito e pouco estudado. Considera-se que este fungo pode ser um agente oportunista, visto ser encontrado muitas vezes em animais sem sinais clínicos da doença. Desta forma, situações tais como muda, grande produção de ovos, alterações ambientais e de higiene, ou qualquer falha na criação que leve ao estresse e imunossupressão, podem propiciar a proliferação do agente e, portanto, ao aparecimento dos sinais clínicos da enfermidade.

\section{Referências Bibliográficas}

Anderson, N. L. 1993. Candida/megabacteria proventriculitis in a lesser sulphur-crested cockatoo (Cacatua sulphurea sulphurea). Journal of the Association of Avian Veterinarians, 7, 197-201.

Bradley, A., Yasuka, H. \& Phalen, D. N. 2005. Macrorhabdus ornithogaster: inhibitory drugs, oxygen toxicity and culturing from feces. USDA sponsored summer research program Athens (GA).

Carrasco, L., Gómez-Villamandos, J. C. \& Jensen, H. E. 1998. Systemic candidosis and concomitant aspergillosis and zygomycosis in two Amazon parakeets (Amazona aestiva). Mycoses, 41, 297-301.

Carvalho, P. R., Queirós, T. S. \& Pita, M. C. G. 2011. Megabacteriose em aves. Pesquisa \& Tecnologia, 8, 1-2.

Cubas, Z. S., Silva, J. C. R. \& Dias, J. L. C. 2014. Tratado de animais selvagens-medicina veterinária. Editora Roca.

Dahlhausen, R. D. 2006. Implications of mycoses in clinical disorders. Clinical Avian Medicine, 2, 691-704.

Filippich, L. J. \& Hendrikz, J. K. 1998. Prevalence of megabacteria in budgerigar colonies. Australian Veterinary Journal, 76, 92-95.

Filippich, L. J., OBoyle, D. A., Webb, R. \& Fuerst, J. A. 1993. Megabacteria in birds in Australian. Australian Veterinary Practitioner, 23, 72-76.

Filippich, L. J. \& Perry, R. A. 1993. Drugs trials megabacteria in budgerigras (Melopsitta undulatus). Australian Veterinary Practitioner, 23, 184-189.

Fraga, C. F. 2014. Ocorrência de doenças micóticas em aves silvestres no Brasil: revisão bibliográfica. Faculdade de Medicina Veterinária. Universidade Federal do Rio Grande do Sul, Porto Alegre, RS.

Fraga, M. E., Medeiros, M. E. S. \& Neves, D. M. 2011. Estudo de Aspergilli durante o período de quarentena de psitacídeos do Centro de Triagem de Animais Silvestres (CETAS) IBAMA, Seropédica, Rj. Revista Brasileira de Medicina Veterinária, 33, 68-72.

Hoppes, S. 2012. Treatment of Macrorhabdus ornithogaster with sodium benzoate in budgerigars (Melopsittacus undulates). Proceedings of the 23rd Annual Conference of the Association of Avian Veterinarians. 
Association of Avian Veterinarians, Seattle (WA).

Kheirandish, R. \& Salehi, M. 2011. Megabacteriosis in budgerigars: diagnosis and treatment. Comparative Clinical Pathology, 20, 501-505.

Lanzarot, P., Blanco, J. L., Alvarez-Perez, S., Abad, C., Cutuli, M. T. \& Garcia, M. E. 2013. Prolonged fecal shedding of 'megabacteria'(Macrorhabdus ornithogaster) by clinically healthy canaries (Serinus canaria). Medical Mycology, 51, 888-891.

Madani, S. A., Ghorbani, A. \& Arabkhazaeli, F. 2014. Successful treatment of macrorhabdosis in budgerigars (Melopsittacus undulatus) using sodium benzoate. Journal of Mycology Research, 1, 21-27.

Martins, N. R. S., Horta, A. C., Siqueira, A. M., Lopes, S. Q., Resende, J. S., Jorge, M. A., Assis, R. A., Martins, N. E., Fernandes, A. A. \& Barrios, P. R. 2006. Macrorhabdus ornithogaster in ostrich, rhea, canary, zebra finch, free range chicken, turkey, guinea-fowl, columbina pigeon, toucan, chuckar partridge and experimental infection in chicken, japanese quail and mice. Arquivo Brasileiro de Medicina Veterinária e Zootecnia, 58, 291298.

Meirelles, C., Cunha, F. S., Davies, Y. M., Guimarães, M. B. \& Ferreira, A. J. P. 2015. Ocorrência de Macrorhabdus ornithogaster em calopsitas (Nymphicus hollandicus) atendidas no ambulatório de aves fmvz-usp entre 2010 e 2014. Revista de Educação Continuada em Medicina Veterinária e Zootecnia, 13, 57-57.

Munhoz, L. S., Finger, P.F., Siedler, B.S., Fischer, G., Hubner, S.O., Sallis, S.E. 2008. Presença de macrorhabdus ornithager em canários belgas (Serinus canarias) oriundos da cidade de Pelotas - Rio Grande do Sul. In; Congresso Brasileiro de Medicina Veterinária, 35, 491492.

Nouri, M. \& Kamyabi, Z. 2010. Occurrence of ventricular candidiasis in a lovebird (Agapornis fischeri). The Iranian Journal of Veterinary Science and Technology, 2, 51-56.
Phalen, D. N. 2007. Diagnosis and management of Macrorhabdus ornithogaster (formerly megabacteria). Veterinary Clinics of North America: Exotic Animal Practice, 8, 299-306.

Phalen, D. N. 2014. Update on the diagnosis and management of Macrorhabdus ornithogaster (formerly megabacteria) in avian patients. Veterinary Clinics of North America: Exotic Animal Practice, 17, 203-210.

Queirós, T. S., Carvalho, P. R. \& Pita, M. C. G. 2011. Megabacteriose: Macrorhabdus ornithogaster em aves - Revisão. PUBVET, Londrina, v. 5, n. 13, p. 1080. PUBVET, 5, 1080.

Scanlan, C. M. \& Graham, D. L. 1990. Characterization of a gram-positive bacterium from the proventriculus of budgerigars (Melopsittacus undulatus). Avian Diseases, 34, 779-786.

Silva, T. M., Okamoto, A. S., Smaniotto, B. D., Pavan, L. F. \& Andreatti Filho, R. L. 2014. Associação de megabacteriose, aspergilose e candidíase em periquitos australianos (Melopsittacus undulatus) em cativeiro, Marília, SP-Relato de caso. Revista Brasileira de Ciência Veterinária, 21, 101-104.

Vasconcelos, T. C. B., Longa, C. S., Albuquerque, D. D. A., Costa, C. H. C. \& Bruno, S. F. 2011. Aspectos clínicos e anatomopatológicos de aspergilose e candidíase em calopsita (Nymphicus hollandicus): relato de caso. Revista Portuguesa de Ciencias Veterinarias, 110, 109-112.

Werther, K., Schocken-Iturrino, R. P., Verona, C. E. S. \& Barros, L. S. S. 2000. Megabacteriosis occurrence in budgerigars, canaries and lovebirds in Ribeirao Preto region-Sao Paulo state-Brazil. Revista Brasileira de Ciência Avícola, 2, 183-187.

\section{Article History:}

Received 20 April 2017

Accepted 8 May 2017

Available on line 11 June 2017

License information: This is an open-access article distributed under the terms of the Creative Commons Attribution License 4.0, which permits unrestricted use, distribution, and reproduction in any medium, provided the original work is properly cited. 